\title{
Censo en Bolivia: apuntes para la construcción de un indicador de multilocalidad $^{1}$
}

\section{Census in Bolivia. Notes for construction of a multi-locality indicator}

\author{
Víctor Hugo Perales Miranda ${ }^{2}$, Marcelo Gonzalo Párraga Guachalla ${ }^{3}$ y \\ Jhosep Luis Usnayo Sirpa ${ }^{4}$
}

Resumen: se hace un recorrido por los censos en Bolivia; revisamos los puntos de discusión y también las críticas sobre la problemática de multilocalidad, planteadas en columnas de opinión de medios de prensa. Luego, se revisa las definiciones de migración interna y un breve estado del arte de trabajos afines a la multilocalidad. Más adelante, se revisan boletas censales de los años 1992, 2001 y 2012. Finalmente, se reflexiona conceptualmente y se brindan pistas para la construcción de un índice de multilocalidad.

Palabras clave: multilocalidad, control vertical de pisos ecológicos, migraciones internas, censo, archipiélago vertical

Abstract: running through Bolivian censuses, we review points of debate and criticisms of the problem of multiple residence, proposed in the opinion columns of print media. We then revise the definitions of internal migration, with a brief state of the art related to multilocality, and review the census formats of 1992, 2001 and 2012. Finally, we provide a conceptual reflection to provide for the construction of an index of multilocality (multiple residence).

Keywords: multi-locality, vertical control of ecological levels, internal migration, census, vertical archipelago

1 Declaramos no tener algún tipo de conflicto de intereses que haya influido en nuestro artículo. 2 Sociólogo. Carrera de Sociología, Universidad Mayor de San Andrés (UMSA), La Paz. E-mail: victorhugo76@gmail.com. orCID: https://orcid.org/0000-0001-5249-1188.

3 Estudiante de Sociología (UMSA), La Paz. E-mail: mgparraga@umsa.bo, orciD: https:// orcid.org/0000-0002-3193-4530.

4 Estudiante de Sociología (UMSA), La Paz. E-mail: jhosepluis.777@gmail.com, orcID: https://orcid.org/0000-0002-4293-1300. 


\section{INTRODUCGIÓN}

El Censo Nacional de Población y Vivienda se llevará a cabo el 16 de noviembre del 2022, como lo indica el Decreto Supremo No 4546 del 21 de julio de 2021. Por ello, hay la necesidad de que se tomen en cuenta algunas lecciones aprendidas extraídas de la ejecución de los últimos censos practicados en Bolivia. Esto implica, además, incorporar las formas como se ha empleado el procesamiento y análisis de datos, tan importantes para la formulación, ejecución y evaluación de políticas públicas, materializadas en planes, programas y proyectos. Algunos expertos, como el sociólogo René Pereira, vienen advirtiendo la necesidad de conjurar algunos problemas que se presentaron en los anteriores censos, en particular el realizado el 2012: la elaboración de la cartografía con información imprecisa o incorrecta, las consabidas disputas de límites geográficos; así como evitar el incentivo del súbito acarreo masivo de personas a sus municipios de origen, pues los criterios de asignación de recursos del presupuesto general están bajo parámetros demográficos (Vacaflor, 2021).

Mediante el presente trabajo, hacemos un breve recorrido por los censos practicados en Bolivia, revisamos los puntos de discusión en algunas revistas nacionales que han dado espacio al debate sobre los censos y también intentamos recoger algunas voces que abordan esta problemática, a través de las columnas de opinión de diarios nacionales. Posteriormente hacemos un recorrido por las definiciones de migración interna y un breve balance del estado del arte de trabajos que están en sintonía con el concepto de multilocalidad, que es el centro de este trabajo. Después revisamos las posibilidades de incorporar un índice de multilocalidad; para ello, hacemos una revisión de las boletas censales de los años 1992, 2001 y 2012. Finalmente, se hace una reflexión sobre el concepto de multilocalidad y se brindan algunas pistas metodológicas para la construcción de un índice de multilocalidad que capte la residencia múltiple, en caso de que así lo practiquen los ciudadanos bolivianos, para los efectos de entender la real magnitud con la que se manifiesta este fenómeno. El resultado del presente trabajo surge de los objetivos del Seminario "Práctica de aplicación de metodologías cuantitativas", de la Carrera de Sociología de la Universidad Mayor de San Andrés. 


\section{LOS CENSOS EN LA HISTORIA DE BOLIVIA}

Bolivia ha tenido hasta el momento once censos. Los dos primeros se realizaron durante el gobierno del Mariscal Andrés de Santa Cruz: en 1831, se registró a 1.088 .768 personas, mientras que el de 1835 empadronó a 1.060.777. Vale mencionar que estos censos se realizaron antes de que se gobernara en el marco de la Confederación Peruano-Boliviana. Durante el siglo XIX, se hicieron cinco censos de población; a los ya mencionados, se suman el censo de 1845, en el que se registraron 1.378.896 habitantes; en 1854, se alcanzó a 2.326.126; mientras que, en el censo de 1882, a poco de haber culminado la Guerra del Pacífico, con el subsecuente desmembramiento territorial, el registro de población alcanzó a 1.172.156 habitantes, en el que también se evidenciaba un decrecimiento demográfico. En el siglo XX, se realizaron cuatro censos: en 1900, cuando se empadronó a 1.766.451 personas; en 1950, cuando se contabilizaron 2.704.165 habitantes; en el de 1976 fueron 4.613 .419 bolivianos; mientras en el año 1992 se registraron 6.420.792. En lo que va del siglo XXI, ya se han realizado dos censos: el del 2001, cuando se llegó a 8.274.325 habitantes y el Censo del 2012, cuando se registró a 10.059.856 personas (Instituto Nacional de Estadística, INE, 2015: 11). Cabe mencionar que desde el año 1992 hay el compromiso de realizar un censo de población y vivienda cada diez años, por lo que ya se ha emitido el Decreto Supremo No 4546 para que el siguiente censo, que vendría a ser el décimo segundo, se realice el 16 de noviembre del 2022 (figura 1).

\section{¿QUÉ DICEN LOS ESTADÍSTICOS}

\section{Y DEMÓGRAFOS SOBRE LOS CENSOS?}

Bolivia tiene solo dos carreras de Estadística en el sistema universitario boliviano, hablamos de la Universidad Mayor de San Andrés de La Paz (UMSA) y la Universidad Autónoma Tomás Frías de Potosí (UATF), mientras que la Universidad Autónoma Gabriel René Moreno (UAGRM) tiene un título de Técnico Superior en Estadísticas Económicas, como título intermedio de la Carrera de Economía de esa universidad. Hay solo dos centros afiliados a las universidades públicas que realizan investigaciones, en cuyo rótulo se inscribe la especialidad de las estadísticas en Bolivia, éstos son: el Instituto de Estadística Teórica y Aplicada (IETA), de la Carrera de Estadística de 
Figura 1. La población en Bolivia en 11 censos

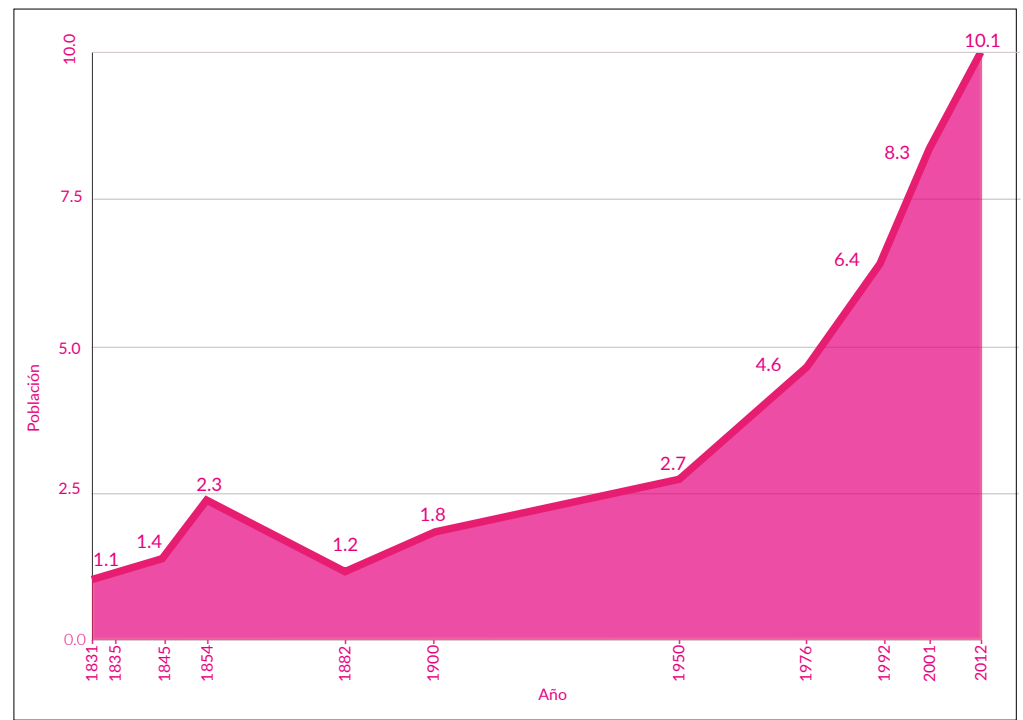

Fuente: Elaboración propia a partir de INE (2015: 11).

la UMSA, y el Centro de Estadísticas Aplicadas (CESA), ligado a varias facultades de la Universidad Mayor de San Simón (UMSS) -lo que no implica que otros centros de investigación también apliquen las estadísticas como una de las formas de desarrollo de sus trabajos. No obstante, de estas dos instituciones, la única que se pronuncia de manera sistemática sobre los censos de población, desde el 2001, ha sido el IETA, mediante su revista, Varianza. Por ese motivo, hacemos a continuación un recuento de las opiniones, críticas, sugerencias y reflexiones en torno al censo, dado que se trata de un referente académico cuya voz es relevante atender.

El nacimiento de la revista Varianza se produce en el ya lejano 2001. En aquella oportunidad, un artículo, a manera de opinión editorial del instituto que promueve la revista, hace una enunciación rápida de los censos del siglo XX: de 1900, 1950, 1976 y 1992 (IETA, 2001). Luego, hace un análisis un tanto más detallado de las dificultades y deficiencias de los censos de 1976 y 1992, que se sugiere que sean superadas en el censo del 2001. Finalmente, 
señala, como tres tareas importantes del censo, la del plan de empadronamiento, coincidente con una actualización cartográfica; la elaboración del cuestionario censal y el alto costo de oportunidad, porque se declararía feriado nacional el día que se realizara el censo del 2001.

Rivero Suguiura (2011) apuntaba algunas sugerencias para la buena marcha del censo de población y vivienda que se llevaría a cabo un año después, es decir, el 2012. Señalaba que el éxito del censo estaba basado en la actualización cartográfica, que implica actualizar planos y mapas, así como datos sobre las áreas geográficas y de los centros poblados, que permitan diseñar de manera óptima la logística para desarrollar el censo. Así también indicaba que los resultados de este censo sirvan para planificar las encuestas de hogares durante los diez años siguientes. Sin embargo, Rivero advirtió que esta tarea principal estaba siendo amenazada por una comunicación que se propaló en el año 2001 sobre las consecuencias del censo, que estaban relacionadas con la redistribución de recursos presupuestarios. Esto, en los hechos, ocasionó el lanzamiento de facto de una especie de "política de migración poblacional momentánea", que implica que algunos municipios ejerzan presiones para que pobladores de las ciudades de origen campesino retornen a sus comunidades solo por uno o dos días, para que sean censados en su lugar de origen. Esta situación alteraba toda la logística y los objetivos de los censos, así como la planificación de las posteriores encuestas de hogares.

El IETA (2012) hace una serie de argumentaciones en torno al censo; señala que si bien éste se asume como parámetro para definir la distribución presupuestaria, la cantidad de escaños de diputados y la planificación de las futuras campañas electorales, éstos no son los objetivos de un censo; porque su perfil es netamente estadístico y sirve para realizar comparaciones con censos anteriores y con los de otros países para el estado de cada una de las variables que forman parte, así como para ajustar los criterios muestrales para la realización de las encuestas de hogares en los siguientes diez años. Pero, además, el hecho de que la población asuma de manera errónea el empleo del censo tiene que ver con un criterio político que ha llevado a que el censo se distorsione al provocar la movilización de muchas personas que se ven amenazadas por la continuidad de sus propiedades en sus lugares de 
origen en áreas rurales y que, por tanto, se ven obligadas a ir puntualmente a censarse en las zonas rurales y no en las ciudades, donde viven permanentemente. Los municipios y las comunidades rurales hacen estas exigencias para contar con más dinero de la redistribución presupuestaria. En tal sentido, lo que sugiere el IETA es centrarse en aspectos técnicos tales como la actualización cartográfica y la disposición del personal de empadronadores suficientes para encarar esta tarea. Deja en claro que no deben politizarse ni tergiversarse los verdaderos objetivos de un censo.

En el número diez de la revista Varianza, el IETA (2013) emitió un pronunciamiento, haciendo recuerdo lo afirmado en los números 8 y 9 de esa revista, en relación al censo de población y vivienda. En particular, se criticó la improvisación del censo, que no cumplió con actualizar la cartografía, lo cual ocasionó serias consecuencias adversas con el nuevo censo; éstas son: la obtención de información censal pero no real, la ausencia de un marco muestral para las encuestas de hogares que se hagan del 2012 hacia adelante; la proyección de población negativa y aleatoria, como La Paz - que en teoría hacia el 2021 o 2022 pasaría a ser la cuarta ciudad de Bolivia por debajo de Santa Cruz de la Sierra, El Alto y Cochabamba, en parte, por las distorsiones que ocasiona la migración momentánea que fue alentada, tal como se indicaba en los números 8 y 9 de la revista Varianza-; así como resultados de población de municipios sin que se tomen en cuenta los nuevos municipios creados, poniendo como ejemplo a municipios que resultaron de la fragmentación de los municipios de Achacachi y Ancoraimes.

Luego, se registran un par de artículos más en la revista Varianza relacionados a los censos de población. Estos dos son de carácter más técnico y ya no están orientados a pronunciarse de manera genérica sobre cuestiones del censo, sino más bien a fortalecer el diseño muestral para aplicarse a las encuestas de hogares en el periodo decenal del 2002 al 2012; en un artículo se establecen ponderaciones y definiciones que lleven a optimizar la estructuración de los indicadores de pobreza moderada y pobreza extrema, dado que los resultados del Censo del 2012 arrojaron resultados distintos a los proyectados (Chirino Gutiérrez, 2016). El otro artículo está planteado para encontrar las motivaciones y explicar los elementos por los cuales la modificación de la pregunta de los censos de población y vivienda de los años 
2001 y 2012, relacionada con lo que los autores denominan el indicador de "autopertenencia indígena" llevó a que haya una significativa variación del referido indicador, que llegó al 20\% de un censo a otro; para esto realizaron dos encuestas, una dirigida a un grupo de tratamiento y otra a un grupo de control, en las que se hallaron variaciones del 12\%. Lo anterior llevó a concluir que la modificación de las preguntas tendentes a medir el indicador de autopertenencia indígena no son comparables en el tiempo (Chirino Gutierrez, Almeida García y Morales Torrez, 2017).

Además de la revista Varianza, la misma revista Temas Sociales, de la Carrera de Sociología de la UMSA, también brindó un espacio para el abordaje de los problemas que se presentan alrededor de la realización del censo del 2012. En el número 35 de esta revista, René Pereira (2014) publicó un artículo en el que, además de mencionar los consabidos problemas relacionados con la actualización cartográfica, que es presentado como el problema recurrente con el que los censos en Bolivia tienen que atravesar, también hizo mención a otro elemento: el de las disputas o conflictos por límites territoriales entre municipios y departamentos. Asimismo, indicó que el censo del 2012 fue seguido y monitoreado por una larga lista de instituciones, algunas vinculadas a la UMSA, la UMSS y otras organizaciones no gubernamentales.

\section{EL CENSO DEL 2022 EN LOS PERIÓDICOS}

Grebe señala la importancia de los censos, sobre todo porque luego de la emergencia sanitaria por la pandemia del COVID-19 se necesita una línea de base que puede proporcionarla el censo de población y vivienda para tener claro por dónde deben priorizarse las políticas públicas. Pone énfasis en que la preparación del censo debe ser participativa, haciendo recuerdo, en parte de su texto, que algunos censos fueron contaminados por el contexto político; hace mención a un grupo de organizaciones de la sociedad civil que demanda la realización del censo (Grebe, 2021).

Omar Velasco (2021) señala que la realización del censo siempre es materia trascendente de discusión por los efectos políticos y financieros que provoca. Indica que el censo debe ser empleado como una información útil para el rediseño de políticas públicas y no debe centrarse solo en la redistri- 
bución de los recursos del Estado, pues si bien hay municipios o gobiernos autonómicos que se verán favorecidos por el crecimiento poblacional, también habrá entidades territoriales autónomas que se verán desfavorecidas por este procedimiento. Pone en el tapete que la búsqueda de recursos pasa por optimizar la captación de recursos propios, en particular a través de los cobros de impuestos - en especial para los municipios-, mientras que sugiere que, en aras de una genuina autonomía, la discusión por el pacto fiscal debe desprenderse del mero reparto de recursos presupuestarios y centrarse en el trabajo conjunto entre las entidades territoriales autónomas, cuya descoordinación se puso de manifiesto en la emergencia sanitaria.

Por su parte, Zaconeta (2021) indica que el censo, además de mostrar la estructura social, económica y demográfica del país, permite trazar los distintos ámbitos en los que se despliegan las políticas públicas; asimismo, el peso demográfico es empleado como un criterio relevante para la redistribución de escaños en la Asamblea Legislativa Plurinacional. El texto fue elaborado un par de meses antes de que se promulgara el decreto supremo que autoriza la realización del censo de población y vivienda en Bolivia para el año 2022, y el autor asumía que el motivo por el cual no se convocaba a un censo precisamente estribaba en una negativa a variar la composición del Legislativo, dado que pese a que la población urbana en Bolivia representa el 70\% de la población, solo cuenta con un 50\% de escaños. Esta situación, según Zaconeta, beneficia al partido de gobierno. Además, casi de soslayo, y sin entrar en detalles, precisa que otro de los temas latentes a ser abordados es aquel relacionado con el pacto fiscal, es decir, sobre la redistribución presupuestaria entre entidades territoriales autónomas en un contexto complicado, luego de la emergencia sanitaria por la pandemia del Covid-19.

En una columna publicada por Rodríguez (2021), en el periódico El Deber de Santa Cruz, el especialista identifica tres ejes de importancia que arrojará el censo: el primero, referido a la consecución de una actualizada imagen demográfica, económica, social y cultural de Bolivia; el segundo, que deriva del primero, la redefinición de la distribución del presupuesto a partir de esa nueva imagen, lo que también conlleva a un reajuste de las políticas públicas; el tercero, que se refiere a la redefinición del número de escaños. El autor asume que la balanza del nuevo peso demográfico se incli- 
na a señalar que Santa Cruz es el departamento con mayor población; por lo que espera que se hagan reajustes con mayores escaños en la Asamblea Legislativa Plurinacional.

Aguilar (2021) sostiene, entre otra cosas, que lo que hará el censo es detonar una disputa entre las regiones que reclamarán más escaños de diputados -se trata del caso de los diputados plurinominales que se definen de acuerdo al peso poblacional--; su análisis se centra en que la consecuencia de la captación de la nueva estructura demográfica del país hará - tomando en cuenta las proyecciones- que departamentos como Santa Cruz reclamen más escaños de diputados plurinacionales en detrimento de departamentos como Oruro, Potosí y Beni.

La Fundación Jubileo (2021) emitió un comunicado en el que, desde su posición de institución que forma parte de la sociedad civil, acoge la medida oficial de llevar adelante el censo. Señala que esta tarea conlleva una serie de pasos previos que deben realizarse, como la actualización de la cartografía; sugiere que para la inclusión de las preguntas en las boletas censales se tomen en cuenta a las instituciones de la sociedad civil y universidades que pueden colaborar en dicha tarea; otro punto es la necesidad de la capacitación para el levantamiento fidedigno de datos.

\section{CONGEPTOS DE MIGRACIÓN INTERNA SEGÚN CELADE}

$\mathrm{Al}$ identificarse recurrentemente que los desplazamientos migratorios durante las fechas próximas al censo tienden a distorsionar los resultados del mismo, y tomando en cuenta que estos desplazamientos los realiza, por lo general, cierta población que ha sido parte de migraciones internas, pasemos a revisar este fenómeno desde la perspectiva conceptual. El Centro Latinoamericano y Caribeño de Demografía (CELADE), que es la División de Población de la Comisión Económica para América Latina y El Caribe (CEPAL), pone a disposición de cualquier persona la base de datos de los flujos de migración interna entre la División Administrativa Mayor (DAM) y la División Administrativa Menor (DAME) de cada uno de los países miembros de la CEPAL en su portal de internet (CELADE-CEPAL, 2021). En el referido sitio web advierte que emplea dos indicadores de migración interna: Migración Reciente, que se evidencia teniéndose en cuenta el cálculo 
de residencia con anterioridad a los cinco años del levantamiento de datos censal; Migrante de toda la Vida, que es quien permanece en un lugar que no es el de su origen, por más de cinco años, también tomándose como referencia el momentos del levantamiento de datos del censo (CELADE-CEPAL, 2021).

Como puede verse, estos indicadores de uso permanente dentro de CELADE permiten además hacer la comparación del estado de la migración interna dentro del país, tomándose como parámetros lugares y momentos y permiten comparar el desarrollo de la migración interna de Bolivia con el de otros países de la región. No obstante, si bien tiene las ventajas de la posibilidad de comparación en el tiempo y lugar o lugares -ya sea dentro del país o comparándose con otros países de la región-, tiene como desventaja el hecho de que no capta la residencia múltiple, la doble residencia, es decir, la multilocalidad. Esta situación explica por qué las boletas censales analizadas en este mismo texto no cuentan con este tipo de preguntas.

Es evidente que se asume que los fenómenos de movilidad humana implican un punto de origen y un punto de destino, sin que haya la posibilidad -en esta lógica conceptual- de tener como domicilio dos lugares distintos y distantes de manera simultánea. Se entiende que desde un enfoque centrado en los individuos hay dificultades para intentar captar los procesos de movilidad que involucren dos lugares que simultáneamente son domicilios de una persona, porque sería asumir que lindan con la facultad de ubicuidad u omnipresencia. Pero, por ejemplo, en Finlandia, donde se estudia el fenómeno de la multilocalidad, a partir de concebir como unidad de análisis a los individuos, resolvieron la medición construyendo un indicador que capta los movimientos migratorios estacionales (Lehtonen, Muilu y Vihinen, 2019). Las características sociales, económicas, políticas y culturales de muchos de los habitantes de las áreas rurales de Bolivia nos conducen a pensar que, si bien podría ser útil decantarse por una solución de captar la multilocalidad centrándose en los individuos, como la que se está aplicando en Finlandia, estos esfuerzos deben apuntar a incluir como unidad de análisis a los hogares, a la familia - y quizás el reto no solo sea pensar en una familia nuclear, sino incluso en una familia extendida, si amerita el caso. Pues hay indicios de que el desarrollo de la multilocalidad en Bolivia se inscribe dentro del contexto de estrategias familiares. 
La incorporación de preguntas en la boleta censal, tendentes a desarrollar un indicador de multilocalidad, en cierta forma podrían también conjurar esa política de migración momentánea (Rivero Suguiura, 2011) que, de facto, se ha expresado en los últimos dos censos de población y vivienda, puesto que se instrumentaliza la información que tiene como base la desestimación de estos criterios de movilidad humana que se evidencian y expresan en las prácticas cotidianas de muchas familias bolivianas, ya sea para pugnar por más escaños de diputados plurinominales o por más recursos presupuestarios de la torta fiscal. De hecho, las críticas a las migraciones momentáneas, que no son pocas, como ha podido observarse en el recuento previo de este trabajo, en realidad no necesariamente revelan un fraude, dado que quienes retornan a sus comunidades de origen en el momento censal no es que solo van a sus comunidades exclusivamente para esa fecha o para elecciones, sean generales o subnacionales, sino que también efectúan esos desplazamientos en distintas estaciones del año como parte de las estrategias familiares que despliegan desde hace mucho. Captar en el censo la multilocalidad permitirá comprender el fenómeno, pero sobre todo dimensionar la magnitud en la que está presente en la sociedad boliviana.

\section{BREVE ESTADO DEL ARTE SOBRE LA MULTILOCALIDAD}

El fenómeno de la multilocalidad, su despliegue en el territorio de lo que hoy llamamos Bolivia, es de larga data; este aserto lo demuestran los trabajos de Murra (1975) sobre la propuesta de la teoría del archipiélago vertical, consistente en que los pueblos andinos prehispánicos ejercían dinamismo e influencia en todo un vasto territorio, aunque discontinuo; se trataba de una suma de archipiélagos ubicados estratégicamente en varios pisos ecológicos, que permitían a esos pueblos una circulación de productos para la subsistencia, la misma que fluía en cada uno de los nodos de ese territorio discontinuo. También son clásicos los hallazgos sobre el mismo tema, realizados por Ramiro Condarco (1987), quien denominó a este fenómeno como "simbiosis interzonal".

El concepto de multilocalidad entraña una o varias estrategias empleadas por familias - en realidad una trama compleja de una familia extensa que articula, pero que también se diferencia, con familias nucleares del mismo 
tronco- que territorializan distintos puntos geográficos entre los que distan decenas o centenares de kilómetros. Así también se encuentran, en algunos casos, en distintos pisos ecológicos o se intercala el desarrollo de actividades en espacios urbanos y rurales, en el marco de una territorialidad discontinua que se asemeja al planteamiento del archipiélago vertical de John Murra (1975) o al de la complementariedad ecosimbiótica de Ramiro Condarco (1987).

El fenómeno del control vertical de pisos ecológicos o de complementariedad ecosimbiótica ha sido vastamente estudiado en la década de 1970 en adelante, en principio, como una característica de las poblaciones que se desplazaban fluidamente entre puntos ubicados en áreas altiplánicas, valles interandinos, áreas tropicales hasta las costas andinas. Esta fluidez, al parecer, nunca se detuvo, como dan cuenta los trabajos de Sánchez Albornoz (2020), quien detectó que, en tiempos de la colonia, dichas migraciones internas en sí se trataban de estrategias para eludir trabajos forzados y el pago de impuestos de los que fueron denominados como forasteros. En estas migraciones, en cierta forma, no desaparecían los contactos y desplazamientos hacia las tierras de origen, a través de diversas tácticas que permitieron que las áreas altiplánicas no tengan una merma demográfica y facilitaran su avituallamiento con productos venidos de áreas del valle, como el maíz, y de áreas tropicales, que aportaban, entre otros productos, la hoja de coca.

Durante el siglo XIX, el trajín y el arrieraje que aparecen en la iconografía de María Melchor Mercado, analizados por Silvia Rivera (2015: 38-39) parecen dar cuenta nuevamente de estos flujos multilocales. Obviamente, si bien podemos detectar la dinámica multilocal en estas épocas, no podemos cerrar los ojos al hecho de que el fenómeno se desenvuelve en contextos globales, socioeconómicos y políticos completamente distintos (figura 2). 
Figura 2. Lámina iconográfica de María Melchor Mercado, llameros challapateños

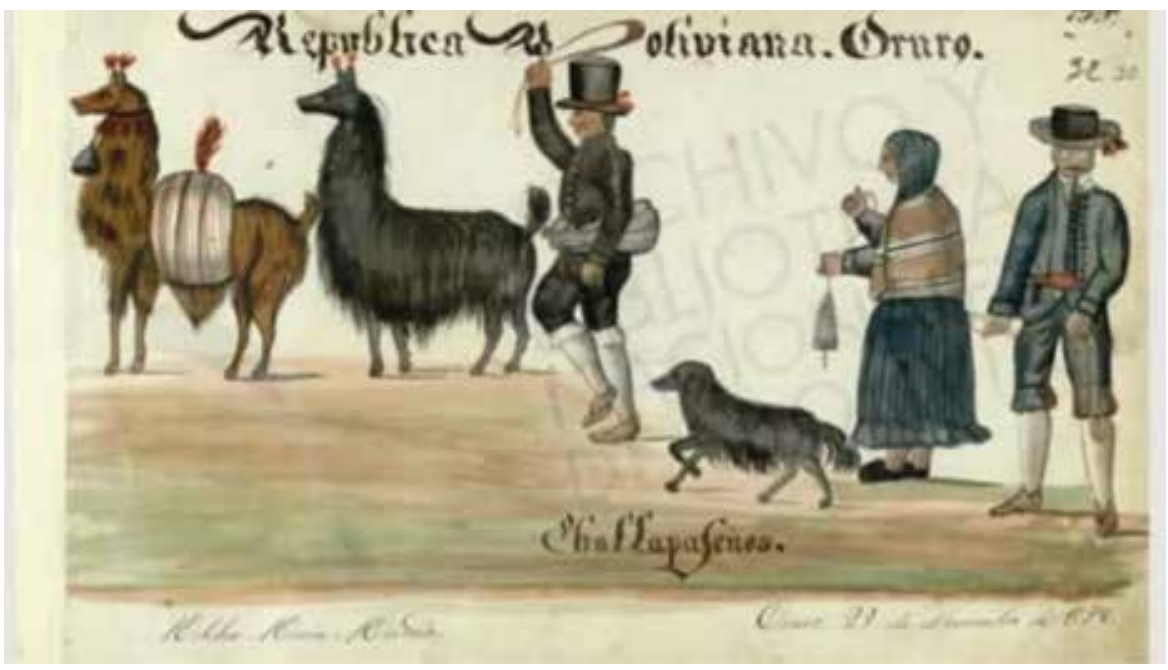

Esta historia tampoco se detuvo en la época republicana ni a comienzos del siglo XX, cuando el régimen hacendatario no solo se inscribió como una política confiscatoria de tierras y exaccionadora de la fuerza de trabajo de la población sometida bajo el régimen de servidumbre, sino que ejerció un control de los cuerpos y de los desplazamientos de aquellas personas convertidas en pongos, lo que se manifestó como tendencia, mas no como una hegemonía absoluta. Prueba de ello son los desplazamientos de los que dan cuenta los trabajos desarrollados sobre el movimiento de los llamados caciques apoderados (Rivera Cusicanqui, 2010; Gotkowitz, 2011), a los que podría sumarse la actividad del arrieraje entre áreas de cultivos agrícolas y su conexión con las minas o también los desplazamientos de algunos comunarios de ayllus hacia las minas para vender su fuerza de trabajo, tal como queda retratado en la novela Tempestad en la Cordillera, de Walter Guevara Arze (1988). Éste, si bien se trata de un retrato con carga ficcional, permite dimensionar las dinámicas de migración interna de la época anterior a la Revolución de 1952.

En los trabajos de Danilo Paz Ballivián (1983: 91-106), podemos advertir que el fenómeno de la multilocalidad probablemente se engarzó 
dentro de la política de colonización que se alentó desde la década de 1960 hacia zonas de Santa Cruz, el Chapare o Alto Beni. Este proceso no cesó hasta entrada la década de 1980 (Bolivia. Ministerio de Planeamiento y Coordinación; UNFPA, 1984), aunque también el fenómeno de multilocalidad tuvo matices en esa época, algunos de ellos recuperados por la icónica obra ochentera de Albó, Greaves y Sandoval (1983), cuyo tomo III lleva el sugestivo título Cabalgando entre dos mundos.

En la década de 1990 y entrados en la primera década el siglo XXI, también se escriben múltiples trabajos que dan cuenta del fenómeno de los "residentes", como se llama a las personas que se han afincado en las ciudades pero que mantienen un contacto fluido y vivaz con sus comunidades de origen, que es la forma como identificamos que se sigue desplegando el fenómeno de la multilocalidad (Spedding y Llanos Layme, 1999; Quispe López y otros, 2002; Llanos Layme, 1999). También podemos recordar la alusión que hace Silvia Rivera (1999) a Simón Yampara, mientras éste fuera Director del Instituto Nacional de Colonización y ministro en la década de 1980: habiendo tenido experiencias en la niñez de migración itinerante, intentó acoplar, aunque infructuosamente, los criterios de doble tenencia de tierra en múltiples pisos ecológicos a las políticas públicas.

En la década de 2010, también se presentan múltiples trabajos que hacen referencia a lo que nosotros identificamos como multilocalidad; de hecho, las reflexiones cada vez son más sesudas y específicas, llámese multilocalidad o residencia múltiple (Spedding Pallet, 2012; Jorgensen, 2011; Villegas Gálvez, 2012). Incluso, en esa década, un trabajo de Xavier Albó (2012: 42-44) reclamaba la necesidad de preguntas en la boleta censal referidas a la doble residencia, la que concebimos como multilocalidad. Agregamos que, si bien existen muchísimos casos de doble residencia, también los hay de residencia múltiple, hasta en más de tres lugares simultáneos. Estas reflexiones sobre la multilocalidad se cierran momentáneamente con el trabajo etnográfico de Nico Tassi y María Elena Canedo (2019), donde los autores conjugan el concepto de multilocalidad con el de multiactividad, a partir de dos estudios de caso donde analizan las articulaciones de los comunarios de Belén, en el municipio de Achacachi, provincia Omasuyos, y Khola, del municipio de Sapahaqui, provincia Loayza, con las ciudades de La Paz y El Alto. 


\section{LA MULTILOCALIDAD EN LAS BOLETAS CENSALES}

DE 1992, 2001 Y 2012

En las boletas censales de 1992, 2001 y 2012, se evidencia una tendencia de incorporación de términos como "solo para el área rural dispersa", ya que de alguna manera entraría en debate qué es lo que sería una población rural o urbana en esos tiempos. Otra cuestión que se ve es la pregunta: “¿quiénes pasaron la noche anterior al día del censo en este hogar?” y también la pregunta “¿dónde vive habitualmente?”, ya que estos ámbitos delimitarían de alguna manera si la persona vive en dos o más lugares, con lo que se podría definir si el encuestado vive en otro sitio diferente de donde se encuentra en el momento del censo; dicho en otras palabras: de dónde viene para hacerse censar.

También se nota la importancia que tiene nacer y vivir de manera habitual en un lugar, ya que en las boletas censales se resaltan y ocupan gran parte de preguntas de la cartilla censal para poder delimitar esas cuestiones. La identidad incorporada como concepción individualizada parte desde el nacimiento hasta la residencia en un sitio. Estos parámetros de medición nos muestran que la población se traslada de un lado a otro, de modo muy diferente de la migración "definitiva"; es decir, se despliega una migración estacional debida a varios factores; entre los más importantes, podemos considerar a los ciclos agrarios.

Sobre la multilocalidad, no se detectan en las boletas censales preguntas específicas, pero las preguntas sobre población existentes sirven para el análisis del comportamiento de este fenómeno, aunque combinadas con algún acotado número de preguntas que deberían agregarse.

En Bolivia, se ve muchas dinámicas sociales que nos hacen ver que cada individuo se mueve y se dedica a numerosas actividades en lugares diferentes con actores sociales distintos. Esto es lo que construye la variedad de residencias de cada individuo; de esta manera, algunos se convierten en residentes, y tienen viviendas y familia en muchos lugares donde van rotando su residencia durante el año.

En las boletas censales de 1992 hasta el 2012, se ve una similitud en el contenido y las preguntas. Algunas preguntas nos pueden mostrar el traslado y la movilidad humana: ¿quiénes pasaron la noche anterior al día del censo en este hogar?, ¿dónde vive habitualmente? o ¿dónde vivía hace 5 años? Las 
respuestas podrían indicar si la persona censada tuvo o tiene una residencia adicional al lugar del empadronamiento.

Las limitaciones de las convenciones sobre multilocalidad atraviesan dificultades; principalmente por las definiciones teóricas, por el momento imprecisas y en debate continuo; pero pequeños avances han permitido algunos progresos. Por ejemplo, en Perú, desde 2017, se ha diseñado el censo para cuantificar a los residentes móviles sobre las variables de captura: educación y trabajo; esto implicó llegar a un nivel de diseño cartográfico distrital y contar con una interesante batería de indicadores sobre movilidad cotidiana de residentes y no residentes; pero aún no se tiene avances para captar la multilocalidad.

En Bolivia, las boletas censales de las últimas rondas se han enfocado en capturar los movimientos migratorios; las preguntas son convencionales en cuanto a las sugeridas por organismos internacionales, pero lamentablemente insuficientes para el levantamiento de información sobre multilocalidad (figura 3). La residencia habitual por sí misma es insuficiente; por ello, se requiere generar relacionamientos con otros elementos, además de contar con un diseño claro sobre los parámetros fundamentales de definición distancia, tiempo y estacionalidad, y éstos a su vez por variables de captura.

Figura 3. Comparación de algunas preguntas en las boletas censales de 1992, 2001 y 2012.

Boletas censales y de encuestas de hogares 1992, 2001, 2012

\begin{tabular}{|l|l|l|}
\hline $\begin{array}{l}\text { Censo Nacional de } \\
\text { Población y Vivienda 1992 }\end{array}$ & $\begin{array}{l}\text { Censo Nacional de } \\
\text { Población y Vivienda 2001 }\end{array}$ & $\begin{array}{l}\text { Censo Nacional de } \\
\text { Población y Vivienda } 2012\end{array}$ \\
\hline $\begin{array}{l}\text { ¿Cuál es el nombre y ape- } \\
\text { llido de todas las personas } \\
\text {-incluyendo niños y recien } \\
\text { nacidos- que pasaron la } \\
\text { noche anterior al día del } \\
\text { censo en este hogar? }\end{array}$ & $\begin{array}{l}\text { ¿Cuántas personas dur- } \\
\text { mieron en esta vivienda la } \\
\text { noche anterior al día del } \\
\text { censo, incluyendo recién } \\
\text { nacidos? }\end{array}$ & $\begin{array}{l}\text { En esta vivienda, ¿cuántas } \\
\text { personas pasaron la noche } \\
\text { anterior al día del censo? } \\
\text { (No olvide incluir a niñas, } \\
\text { niños, recién nacidos, ancianas } \\
\text { ancianos) }\end{array}$ \\
\hline
\end{tabular}




\begin{tabular}{|l|l|l|}
\hline $\begin{array}{l}\text { ¿Dónde vive habitual- } \\
\text { mente? }\end{array}$ & $\begin{array}{l}\text { ¿Dónde vive habitualmen- } \\
\text { te? }\end{array}$ & $\begin{array}{l}\text { ¿Dónde vive habitual- } \\
\text { mente? }\end{array}$ \\
\hline $\begin{array}{l}\text { ¿Dónde vivía habitual- } \\
\text { mente hace 5 años? }(1987)\end{array}$ & $\begin{array}{l}\text { ¿Dónde vivía habitualmen- } \\
\text { te hace 5 años? (1996) }\end{array}$ & ¿Dónde vivía hace 5 años? \\
\hline ¿Dónde nació? & ¿Dónde nació? & ¿Dónde nació? \\
\hline
\end{tabular}

Fuente: Elaboración propia a partir de datos del INE.

\section{SOBRE LA MULTILOCALIDAD Y PISTAS METODOLÓGICAS}

\section{DE CÓMO CAPTARLA}

El trabajo de Lehtonen y otros (2019) analiza la multilocalidad como un fenómeno común e intenta superar a la población invisible, resultado de medidas convencionales sobre población. La idea principal del artículo mencionado fue elaborar una política que aproveche el uso de los servicios de salud en Finlandia, considerando el fenómeno de la multilocalidad. Para el autor, la multilocalidad tiene que tener un enfoque rural, por las implicaciones de identidad, pertenencia y, sobre todo, la estacionalidad en los desplazamientos observados; alrededor de 2,2 millones de personas se desplazan entre áreas rurales y urbanas, con una estadía entre los 27 y 72 días del año.

Los desplazamientos se han convertido en una necesidad estructural, en una estrategia de vida, pero a pesar de ser un fenómeno de la cotidianidad de las personas, éstos no son capturados por los hacedores de políticas públicas. Los efectos en los municipios "afectados" en algunos casos generan ingresos extras; en otros, saturan los servicios públicos disponibles, pero la propuesta recoge la posibilidad de rescatar los servicios públicos de salud que se han encontrado centralizados en zonas de alta densidad poblacional. La propuesta se hace interesante al ver los desplazamientos como el eje para reorganizar los servicios de salud, minimizando costos, focalizando servicios y prestaciones. La gran fortaleza de la propuesta es la calidad de información de los desplazamientos poblacionales, cuyas mediciones por tiempo, distancia, estacionalidad y una batería de encuestas de distintos niveles de gobierno permiten "cuadricular" las áreas de desplazamiento. Con los datos se establecen las magnitudes de movimiento, para luego estimar los costos 
de optimizar los servicios con incentivos a los desplazamientos, según los periodos de mayor movilidad.

Los aportes y desafíos detallados en el artículo de Lehtonen y otros (2019) extrapolados a nuestra realidad nos permiten trazar objetivos de política y sobre todo propuestas de diseño; las limitaciones del trabajo en Bolivia pasan, en primer lugar, por la calidad de la información; no se tiene conocimiento sobre qué instancia en algún nivel de gobierno realiza registros de multilocalidad, menos si se cuenta con la posibilidad de levantar datos georreferenciados. Por tanto, estimar magnitudes de población en desplazamiento en el corto plazo no es posible.

El aporte pasa por pensar en el diseño de instrumentos factibles, lo que nos lleva a proponer al menos dos elementos: tiempo y distancia. Se da a entender que la multilocalidad se define para fines cuantitativos mediante estos elementos, siendo el desafío definirlos y estimarlos en nuestro contexto. ¿cuál es la estadía mínima para hablar de multilocalidad en Bolivia?, ¿cómo capturar la multilocalidad en el país cuando está económicamente concentrada en el eje central?, ¿el tiempo de estancia y la estacionalidad responden a motivaciones económicas y también culturales?, ¿cómo cuantificar la magnitud de los desplazamientos?, ¿son estos desplazamientos representativos?

Si se produce un desplazamiento como mecanismo de acceso a servicios públicos (salud, educación, infraestructura), entonces puede presentarse una distorsión en el estado real de la necesidad del proyecto, con riesgos en implementar propuestas sobredimensionadas o subestimadas; en ese sentido, será necesario que para el diseño de proyectos de desarrollo se analice también el desplazamiento poblacional. El fondo de un análisis de multilocalidad podría asentar una idea que políticamente no sería bien vista, y es el tema rural del país: se ha mitificado una Bolivia rural como un capital político, el "limpiar" esta visión puede ser mal entendido y tener efectos en los acuerdos políticos vigentes. Cabe precisar que un interesante campo de análisis para la multilocalidad son los centros interurbanos o nuevas ciudades del país que, por su ubicación geográfica y crecimiento poblacional, son susceptibles de ser espacios que registren estos movimientos de manera más recurrente, además de ser espacios más manejables para la captura de información. 
Es común que en los censos en los cuales se opte por diseños de captura de movilidad poblacional del tipo pendular, tales diseños se centren en los movimientos cotidianos de la población. Es usual encontrar en la revisión bibliográfica definiciones en función de motivaciones laborales; sin embargo, se ha asentado la visión de ampliar las posibilidades del concepto. Así, el movimiento pendular se refiere a los desplazamientos cotidianos para la adquisición de bienes o servicios sin cambio de residencia, motivados por distintos fines (recreativos, laborales, educacionales, etcétera), pero de corta permanencia ${ }^{5}$. Sin embargo, los estudios aplicados por temas de captura de información dan mayor relevancia a los desplazamientos por motivos laborales y educacionales, principalmente, por ser los de mayor recurrencia (Alcântara de Vasconcellos, 2010).

El movimiento pendular es producto de la tendencia de la metropolitización de las ciudades; y sobresale la idea planteada por expertos en urbanidad del siglo XX sobre la ciudad sin límites, donde las diferencias entre área rural y urbana cada vez son más difusas. Hoy en día, asumir solamente variables económicas y sociales para diferenciar ambas es erróneo, debemos pensar en términos de funcionalidad del espacio geográfico para medir y monitorear el espacio de las metrópolis, lo cual solo es posible a partir de la movilidad poblacional (Miralles-Guasch y Cebollada, 2009). En nuestro contexto, solo basta pensar en la aglomeración de personas en los alrededores de la iglesia de San Francisco de la ciudad de La Paz o en las estaciones de la línea amarilla o de la línea morada del teleférico o en esos desplazamientos que se realizan a la Ceja de El Alto.

Si bien la bibliografía es amplia en las explicaciones teóricas sobre las causas de los desplazamientos, como culturales, geográficos, económicos y sociales, las metodologías no han tenido cambios sustanciales; básicamente se centran en diseños de instrumentos censales o de encuestas de hogares que capturen la residencia, el lugar de trabajo y el tiempo de permanencia a un nivel de secciones municipales e incluso a nivel distrital ${ }^{6}$. Una reciente y exitosa experiencia fue la de Paraguay en 2012, que, por sus características,

5 Nos referimos a que permanencia implica que existe una repetición constante, como los desplazamientos diarios interurbanos de La Paz a El Alto.

6 En términos más formales, se refiere a la incorporación de la definición de la unidad geográfica de ocupación distinta a la de residencia. 
tomó la variable trabajo como dominante para el diseño de sus instrumentos (DGEEC, 2016).

Con la reciente aparición del big data (datos masivos) y la interconectividad digital de las personas, estamos pasando de aplicar no solamente modelos de gravitación, los cuales ponderan el posible costo en función de la distancia y tiempo del desplazamiento, a refinamientos de seguimiento de tráfico interurbano e incluso a diseños de concentración de señales telefónicas para la construcción de datos que permitan un mapeo de los alcances del desplazamiento cotidiano (Lehtonen, Muilu y Vihinen, 2019); algunos de estos modelos también están siendo empleados en China para mapear qué individuos han ingresado a zonas de riesgo en el contexto de la emergencia sanitaria por el coronavirus (Li y otros, 2021).

De otro lado, la mulitlocalidad es un concepto mucho más amplio; no solo implica movilidad, sino residencia, hogar y configuración familiar (figura 4), se refiere a un fenómeno aún en construcción que engloba la actividad espacial de los individuos y sus grupos. Hay un amplio debate sobre si es o no un fenómeno propio de la globalización, principalmente por la rápida transitabilidad de un espacio a otro, sin que ambos espacios dejen de ser habitados de manera permanente; sobre esto hay un activo debate, que ha hecho de los acercamientos empíricos, imperfectos o confusos (Dittrich-Wesbuer et al., 2015), pues no logra identificarse claramente un punto de origen y otro de destino, ya que se trata de habitar espacios discontinuos de manera simultánea; no se trata de un fenómeno de ubicuidad, sino más bien de disposición de miembros de la familia en los distintos espacios, en distintos momentos, respondiendo la multilocalidad a una estrategia no individual, sino familiar.

En ese sentido, trataremos brevemente de hacer una delimitación del concepto de multilocalidad a partir de los principales elementos planteados, cuyas conjunciones abren variaciones al fenómeno. En un sentido didáctico, la multilocalidad se configura de manera multidireccional; así, se supera la idea de simple movilidad cotidiana planteada anteriormente. Es decir, el desplazamiento y el tiempo de estadía "afecta" a las categorías planteadas, expande los consumos, configura una serie de nuevos capitales, saberes y 
crea estacionalidades de convivencia ${ }^{7}$ (Hamman et al., 2014). Según Gallez y Kaufmann (2009), la estacionalidad es propia de la incidencia de la movilidad de un lugar y su permanencia, donde se crean nuevas estructuras sociales de orden en tiempo, espacio y recursos; la multilocalidad lo trastoca todo, desde la propiedad, hasta las relaciones de parentesco.

Figura 4. Fenómeno de la multilocalidad.

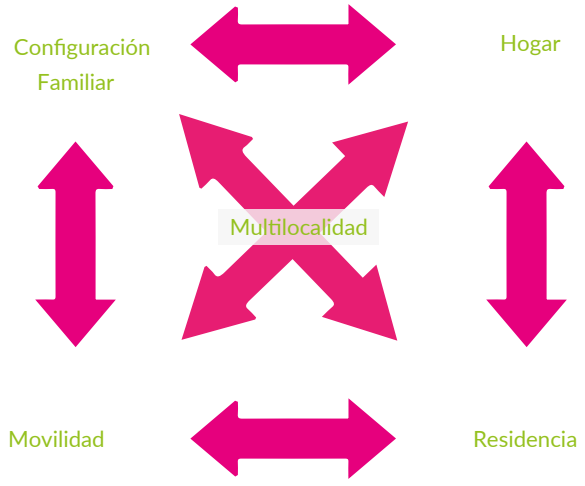

Fuente: elaboración propia.

La multilocalidad es una cotidianidad funcional y activa con prácticas sociales concretas, físicas y presenciales; la movilidad crea nuevas configuraciones familiares en distintos lugares y temporalidades, también sentidos de pertenencia. Así, el hogar pasa de ser un espacio único a ser un espacio multilugar. Estas connotaciones crean un sinnúmero de desafíos al momento de plantear metodologías de medición cuantitativa de la multilocalidad (Hamman, Blanc, Duchêne-Lacroix, Freytag y Kramer, 2014). No hay definiciones metodológicas genéricas; son, de hecho, muy específicas a los contextos y fines de política pública que se buscan. Así, por ejemplo, en Francia se busca hacer visible el tema de la multipropiedad; en Suiza, la

7 Según Kaufmann, la estacionalidad es propia de la incidencia de la movilidad de un lugar y su permanencia (Gallez y Kaufmann, 2009), donde se crean nuevas estructuras sociales de orden en tiempo, espacio y recursos; la multilocalidad trastoca todo: desde la propiedad hasta las relaciones de parentesco. 
multiresidencia; en Finlandia, la prestación de servicios básicos, y cada uno delimita una permanencia que supere una estadía en tiempo distinta.

Los institutos de estadística varían las categorías, según fines de política, en la experiencia de Francia en específico y Europa en general; lo usual ha sido la aplicación de encuestas continuas con diseño de panel, que captura tres elementos: la propiedad, la familia y el trabajo; éstos en función de tres parámetros: distancia, tiempo de estadía y estacionalidad. Con dichos elementos se identifica a un multilocal o a un multiresidente con sus respectivas variaciones. En las investigaciones, destacan estudios con modelos de redes, análisis de multinivel, métodos de estadística espacial y recientemente los place connectivity index ${ }^{8}$ (Hamman, Blanc, Duchêne-Lacroix, Freytag y Kramer, 2014; Li, y otros, 2021) (figura 5).

Figura 5. Componentes de la multilocalidad

\begin{tabular}{|c|c|c|c|c|c|}
\hline Parámetros & Tipo & \multicolumn{2}{|c|}{ Propiedad } & \multicolumn{2}{|c|}{ Familia } \\
\hline \multirow{2}{*}{ Distancia } & \multirow{3}{*}{ Mutilocal } & Sí & No & Sí & No \\
\hline & & \multicolumn{4}{|c|}{ Trabajo } \\
\hline \multirow{2}{*}{ Tiempo de estadía } & & \multicolumn{2}{|c|}{ Sí } & \multicolumn{2}{|c|}{ No } \\
\hline & \multirow{4}{*}{ Multiresidente } & \multicolumn{2}{|c|}{ Propiedad } & \multicolumn{2}{|c|}{ Familia } \\
\hline \multirow{3}{*}{ Estacionalidad } & & Sí & No & Sí & No \\
\hline & & \multicolumn{4}{|c|}{ Trabajo } \\
\hline & & \multicolumn{2}{|c|}{ Sí } & \multicolumn{2}{|c|}{ No } \\
\hline
\end{tabular}

Fuente: Elaboración propia.

\section{CONCLUSIONES}

La importancia de la construcción de un índice de multilocalidad es que los resultados pueden conseguir un factor de equilibrio ante los serios desajustes que ocasiona la súbita movilidad humana en épocas de levantamiento de

8 Significa «Índice de Conectividad de Lugares», una forma de detectar los desplazamientos de los individuos, a partir del monitoreo de aplicaciones de los cada vez más usados smartphones, que además cuentan con la tecnología de rastreo geográfico GPS. 
datos censales, el acarreo de personas asentadas en áreas urbanas hacia localidades rurales, con la finalidad de captar mayores fondos presupuestarios para el municipio, así como también para alcanzar los fondos que respalden el financiamiento de los POA (Planes Operativos Anuales) que se asignan de comunidad a comunidad. De otro lado, en la práctica, cuando se ejecutan programas o proyectos, la falta de pobladores que estaban consignados en el censo se trata de un factor de distorsión de procesos de planificación que perjudican la consecución del VAN (Valor Actual Neto) y el TIR (Tasa Interna de Retorno) inicialmente proyectados, colocando la ejecución de proyectos o programas en peligro de ser declarados inelegibles.

La recurrente disputa del acarreo de personas durante el momento censal, si bien puede entrañar presiones que se inscriben dentro del marco de lo que han denominado "política migratoria momentánea", estas presiones no necesariamente están reñidas con la existencia del mantenimiento de un vínculo fuerte y supérstite de la persona que llega a la comunidad de origen con la finalidad de censarse. De hecho, regresan muchas personas a su comunidad de origen porque el vínculo es vivo; de lo contrario, no habría ningún mecanismo de presión que las autoridades de su comunidad de origen ejerzan hacia este tipo de personas. De hecho, la multilocalidad es la expresión de la vitalidad de la preservación de los vínculos con la comunidad de origen, no solo para preservar el patrimonio privado, sino para escenificar rituales que ponen de relieve el prestigio alcanzado fuera de la tierra de nacimiento ante sus congéneres; así como para participar dentro de una red compleja de circulación de bienes y servicios a los que bien podríamos llamarlos dones, parafraseando a Mauss.

Estas situaciones -las mostradas en el trabajo- nos llevan a reflexionar que si bien se muestra la dificultad por la que hay que transitar para seguir las pistas de la posible construcción del índice de multilocalidad, también este esfuerzo ofrece como compensación la dimensión de su relevancia. De hecho, entender la dinámica social, cultural, económica y política de la multilocalidad podría ser la llave para entender que los procesos de movilidad humana o de migración interna en Bolivia tienden a gestionar el riesgo, diversificando los esfuerzos en múltiples actividades y lugares; pero que también es un rastro que podría llevarnos a entender que el hecho de que 
en la práctica se capten ingresos en un lugar próspero, no necesariamente implica abandonar y reubicar - seguir apostando- parte de esos ingresos en la comunidad de origen, en donde las posibilidades de captar ingresos serían algo más complicadas.

\section{BIBLIOGRAFÍA}

Aguilar, Waldo (25 de julio de 2021). "El censo de 2022 y los mecanismos para la redistribución de escaños". Los Tiempos [sección País]. Recuperado de https://www.lostiempos.com/actualidad/pais/20210725/ censo-2022-mecanismos-redistribucion-escanos

Albó, Xavier (2012). "Censo 2012 en Bolivia: posibilidades y limitaciones con respecto a los pueblos indígenas". T'inkazos, 32, 33-45. Recuperado de http://www.pieb.com.bo/2016/BPIEB/BPIEBT_Tinkazos_32.pdf Albó, Xavier, Greaves, Tomás y Sandoval, Godofredo (1983). Chukiyawu. La cara aymara de La Paz. III. Cabalgando entre dos mundos. Cuaderno de investigación 24, tomo III. La Paz: CIPCA.

Alcântara de Vasconcellos, Eduardo (2010). Análisis de la movilidad urbana: espacio, medio ambiente y equidad. Bogotá: Corporacion Andina de Fomento. Bolivia. Ministerio de Planeamiento y Coordinación y UNFPA (1984). Tras nuevas raices... Migraciones internas y colonización en Bolivia. La Paz: UNFPA. Proyecto de Políticas de Población.

Centro Latinoamericano y Caribeño de Demografía (CELADE). Comisión Económica para América Latina y el Caribe (CEPAL) (agosto de 2021). Base de Datos de Migración Interna en América Latina y el Caribe. Recuperado de https://celade.cepal.org/bdcelade/mialc/

Chirino Gutiérrez, Álvaro (septiembre de 2016). "2 censos, 10 años de encuestas a hogares en Bolivia tiempo de reponderar y definir un diseño muestral comparable para los indicadores de bienestar". Varianza, 12, 25-33.

Chirino Gutierrez, Álvaro, Almeida García, María y Morales Torrez, Bladimir (diciembre de 2017). “¿Reducción de la auto pertenencia indígena en Bolivia? Una aplicación experimental en el diseño de cuestionarios de los censos 2001 y 2012". Varianza, 14, 9-21. 
Condarco, Ramiro (1987). "Simbiosis interzonal". En Ramiro Condarco y John Murra, La teoría de la complementariedad vertical eco-simbiótica (pp. 7-28). La Paz: Hisbol.

Dirección de Estadísticas Sociales y Demográficas (DGEEG) (2016). Migración pendular en el Paraguay, 2012. Asunción: Dirección General de Estadísticas y Censos (DGECG)-República de Paraguay. Recuperado de https://www. ine.gov.py/Publicaciones/Biblioteca/documento/cf82_Migracion\%20 Pendular\%20en \%20el\%20Paraguay.pdf

Dittrich-Wesbuer, Andrea, Kramer, Caroline, Duchene-Lacroix, Cédric y Rumpolt, Peter (2015). "Multi-local Living Arrangements. Approaches to Quantification". Tijdschrift voor economische en sociale geografie, 106(4), 409-424. Fundación Jubileo (21 de julio de 2021). Censo 2022: voluntad Política y corresponsabilidad ciudadana. Recuperado de https://jubileobolivia.org.bo/ download.php? $\mathrm{k}=990$

Gallez, Caroline y Vincent Kaufmann (2009). "Aux racines de la mobilité en sciences sociales". En Mathieu Flonneau y Vincent Guigueno, De l'histoire des transports à l'histoire de la mobilité? (pp. 41-55). Rennes: Presses universitaires de Rennes.

Gotkowitz, Laura (2011). La revolución antes de la revolución: luchas indígenas por tierra y justicia en Bolivia 1880-1952. La Paz: Plural.

Grebe, Horst (25 de abril de 2021). "El próximo censo de población y vivienda". La Razón [sección Voces], p. A25.

Guevara Arze, Walter (1988). Tempestad en la Cordillera. La Paz: Juventud.

Hamman, Philippe, Maurice Blanc Cédric Duchêne-Lacroix, Tim Freytag y Caroline Kramer (2014). Questionner les mobilités résidentielles à l'aune de la multilocalité. Paris: Néothèque. Recuperado de https://www.researchgate. net/publication/265017514_Questionner_les_mobilites_residentielles_a_l'aune_de_la_multilocalite

Instituto Nacional de Estadística (INE) (2015). Censo de Población y Vivienda 2012 Bolivia: características de la Población. La Paz: Instituto Nacional de Estadística. Recuperado de https://bolivia.unfpa.org/sites/default/files/ pub-pdf/Caracteristicas_de_Poblacion_2012.pdf

Instituto de Estadística Teórica y Aplicada (IETA) (noviembre de 2013). “Resultados del Censo de Población y Vivienda 2012". Revista Varianza, 10, 
67-74. Recuperado de http://www.revistasbolivianas.org.bo/scielo.php?script=sci_arttext\&pid=S9876-67892013000100019\&lng=es\&nrm=iso Instituto de Estadística Teórica y Aplicada (IETA) (noviembre de 2012). "El Censo de Población y Vivienda 2012". Revista Varianza, 9, 70-71. Recuperado de http://www.revistasbolivianas.org.bo/scielo.php?scrip$\mathrm{t}=$ sci_arttext\&pid=S9876-67892012000100019\&lng $=\mathrm{es \& nrm}=$ iso Instituto de Estadística Teórica y Aplicada (IETA) (octubre de 2001). “¿Qué podemos esperar del censo 2001?" Varianza, 1, 34-39. Recuperado de http://www.revistasbolivianas.org.bo/scielo.php?script=sci_arttext\&pi$\mathrm{d}=\mathrm{S} 9876-67892001000100010 \& \operatorname{lng}=\mathrm{es} \& n \mathrm{~mm}=$ iso

Jorgensen, Kaylen (2011). 'El 'archipiélago vertical' andino. El control vertical de pisos ecológicos y dinámicas contemporáneas de migración”. En Nelson Antequera Durán y Cristina Cielo, Ciudad sin fronteras: multilocalidad urbano rural en Bolivia (pp. 71-92). La Paz: RITU Bolivia, Gobierno Autónomo Municipal de La Paz, CIDES-UMSA, Fundación PIEB, Oxfam GB y Universidad de California Berkeley.

Lehtonen, Olli, Toivo Muilu y Hilkka Vihinen (2019). "Multi-Local Living - an opportunity for Rural Health Services in Finland?" European Countryside, 11(2), 257-280.

Li, Zhenlong; Xiao Huang, Xinyue Ye, Yuqin Jiang, Yago Martin, Huan Ning, Michael E. Hodgson, y Xiaoming Li (julio de 2021). "Measuring global multi-scale place connectivity using geotagged social media data". Scientific Reports, 11. Recuperado de https://www.nature.com/articles/ s41598-021-94300-7.pdf

Llanos Layme, David (1999). Migración y estructura comunal andina: una aproximación teórica al estudio de la migración y relaciones sociales en el agro andino. La Paz: Instituto de Investigaciones Sociológicas "Mauricio Lefebvre", UMSA. Miralles-Guasch, Carme y Ángel Cebollada (2009). "Movilidad cotidiana y sostenibilidad, una interpretación desde la geografía humana". Boletín de la Asociación de Geógrafos Españoles, 50, 193-216.

Murra, John (1975). Formaciones económicas y políticas del mundo andino. Lima: Instituto de Estudios Peruanos.

Paz Ballivián, Danilo (1983). Estructura agraria boliviana. La Paz: Popular. 
Pereira Morató, René (julio de 2014). "El censo fracasó”. Temas Sociales, 35, 183-200. Recuperado de http://www.revistasbolivianas.org.bo/scielo. php?script=sci_arttext\&pid=S0040-29152014000200007\&lng=en\&n$\mathrm{rm}=$ iso

Quispe López, Eliseo, Alberto Luis Aguilar Calle, Ruth Carol Rocha Grimoldi, Norka Araníbar Cossio, Blanca Huanacu Bustos y Walter Condori Uño (2002). Tierra y territorio: thakhi en los ayllus y comunidades de ex hacienda. La Paz: Fundación PIEB.

Rivera Cusicanqui, Silvia (2015). Sociología de la imagen: ensayos. Buenos Aires: Tinta Limón.

Rivera Cusicanqui, Silvia (2010). Oprimidos, pero no vencidos, 4. ${ }^{\mathrm{a}}$ ed. La Paz: La Mirada Salvaje.

Rivera Cusicanqui, Silvia (1999). "Sendas y senderos de la ciencia social andina". Dispositio, 24(51), 149-169.

Rivero Suguiura, Fernando (noviembre de 2011). "El futuro Censo de Población y Vivienda en nuestro país". Varianza, 8, 69-70. Recuperado de http:/ / www.revistasbolivianas.org.bo/scielo.php?script=sci_arttext\&pi$\mathrm{d}=\mathrm{S} 9876-67892011000100018 \& \operatorname{lng}=\mathrm{es} \& \mathrm{nrm}=$ iso

Rodríguez A. y Antonio Gary (25 de julio de 2021). "¿Por qué es importante el Censo de Población y Vivienda del 2022?". El Deber [sección Sociales]. Recuperado de https:/ /eldeber.com.bo/opinion/por-que-es-importanteel-censo-de-poblacion-y-vivienda-del-2022_240466

Sanchéz-Albornoz, Nicolás (2020). Trabajo y migración en los Andes coloniales. Lima: Banco Central de Reserva del Perú/IEP.

Spedding Pallet, Alison (2012). “¿Acaso las personas son árboles, plantados en un sólo lugar?: crítica conceptual a los estudios sobre "migración"”. Temas Sociales, 32, 187-225.

Spedding, Alison y David Llanos Layme (1999). "No hay Ley para la cosecha": un estudio comparativo del sistema productivo y las relaciones sociales en Chari (provincia Bautista Saavedra) y Chulumani (provincia Sud Yungas), La Paz. La Paz: PIEB/Sinergia.

Tassi, Nico y Canedo, María Elena (2019). "Una pata en la chacray una en el mercado": multiactividad y reconfiguración rural en La Paz. La Paz: CIDES-UMSA. 
Vacaflor, Nancy (28 de mayo de 2021). Investigador de la UMSA pide al Gobierno un decreto supremo que declare de prioridad nacional el censo. Recuperado de Observación ciudadana de la democracia: https://ocdbolivia.org/ notas-comunicacion/investigador-de-la-umsa-pide-al-gobierno-un-decreto-supremo-que-declare-de

Velasco, Omar (01 de agosto de 2021). "Censo 2022, autonomías a medias tintas". La Razón [Suplemento Tiempo Político], p. 3E.

Villegas Gálvez, Helmer B. (2012). "Movilidad humana e itinerarios ruralurbano en el departamento de La Paz". Temas Sociales, 32, 45-59.

Zaconeta Torrico, Alfredo J. (17 de abril de 2021). “¿Por qué el gobierno del MAS no quiere hacer un Censo de Población el 2022?" Rimay Pampa. Recuperado de Rimaypampa: https://rimaypampa.org/por-que-el-gobierno-del-mas-no-quiere-hacer-un-censo-de-poblacion-el-2022/ 